\title{
Aspectos epidemiológicos da Doença de Chagas canina no semiárido paraibano ${ }^{1}$
}

\author{
Rodrigo S. Mendes ${ }^{2 *}$, Vanessa L. Santana², Ana M. Jansen³ ${ }^{3}$ Samantha Cristina \\ C. Xavier ${ }^{4}$, Ivana F. Vidal' ${ }^{2}$, Tereza E.F. Rotondano ${ }^{4}$ e Almir P. Souza ${ }^{2}$
}

\begin{abstract}
Mendes R.S., Santana V.L., Jansen A.M., Xavier S.C.C., Vidal I.F., Rotondano T.E.F. \& Souza A.P. 2013. [Epidemiological aspects of canine Chagas disease in the semi-arid region of Paraiba.] Aspectos epidemiológicos da Doença de Chagas canina no semiárido paraibano. Pesquisa Veterinária Brasileira 33(12):1459-1465. Programa de Pós-Graduação em Medicina Veterinária, Universidade Federal de Campina Grande, Avenida Universitária s/n, Campus de Patos, Bairro Sta Cecília, Patos, PB 58708-110, Brazil. E-mail: rodrigo.souza.mendes@gmail.com

In order to determine the epidemiological aspects involving canine Chagas disease (CD) and to identify major risk factors for the disease in the semi-arid region of Paraiba, a study was conducted in the rural area of Patos/Paraíba, Brazil, where the area was divided into three sampling strata (North, South and West) and, in each stratum we randomly sampled about 294 houses, and all the pet dogs represented the elementary units of study. In each household an epidemiological questionnaire was administered to obtain information on indicators that favor the spread of the disease in peridomestic segment. Serological diagnosis for CD in dogs was based on three methods (IFA, ELISA and HAI), considering positive samples that had at least two tests reagents and absence of cross-reaction. For the rural area, the prevalence of seropositive dogs for Trypanosoma cruzi by sampling stratum was: North $6.05 \%$, South $3.59 \%$ and West $2.97 \%$, corresponding to a total prevalence of $4.08 \%$. The risk factors (odds ratio, OR) were evidenced in multifactorial analysis: wall type $(\mathrm{OR}=2.59$ [1.24-5.4]), presence at warehouse $(\mathrm{OR}=1.89$ [1.31$3.0])$, present at chicken stall $(\mathrm{OR}=8.31$ [1.29-61.7]), contact with animals $(\mathrm{OR}=9.11$ [1.12-73.9]), contact with birds $(\mathrm{OR}=9.7$ [1.81-52.83]), captured triatomines $(\mathrm{OR}=16.58$ [3.43-80.23]) and anthropogenic (OR=4.35 [1.36-14.0]. From the results it was possible to obtain information pertaining to the epidemiological situation of Chagas Disease highlighting biogeographical characteristics of rural semi-arid region of Paraiba, raising dogs and the risk factors disclosed in the highlighted contact with birds and ecotopes operationalizing indicators to be watched and considered in the chain of transmission of the disease in the region.
\end{abstract}

INDEX TERMS: Chagas disease, prevalence, risk factors, rural, peridomiciles, dogs.

\footnotetext{
${ }^{1}$ Recebido em 20 de junho de 2013.

Aceito para publicação em 2 de dezembro de 2013.

${ }^{2}$ Programa de Pós-Graduação em Medicina Veterinária, Universidade Federal de Campina Grande (UFCG), Avenida Universitária s/n, Bairro Santa Cecília, Patos, PB 58708-110, Brasil. *Autor para correspondência: rodrigo.souza.mendes@gmail.com

${ }^{3}$ Laboratório de Referência em Taxonomia e Diagnóstico de Reservatórios Silvestres das Leishmanioses, Instituto Oswaldo Cruz (IOC/Fiocruz), Av. Brasil 4365, Pavilhão Rocha Lima, 5º andar, Rio de Janeiro, RJ 21045900, Brasil.

${ }^{4}$ Departamento de Ciências Biológicas, Universidade Federal de Pernambuco (UFPE), Av. Prof. Moraes Rego s/n, Cidade Universitária, Recife, PE 50670-901, Brasil.
}

RESUMO.- Com o objetivo de determinar os aspectos epidemiológicos que envolvem a doença de Chagas (DC) canina e identificar os principais fatores de risco da enfermidade no semiárido paraibano, foi conduzido um estudo na zona rural de Patos, onde a área foi dividida em três estratos amostrais (Norte, Sul e Oeste) e, em cada estrato foram amostradas aleatoriamente 294 casas, e dessas todos os cães domiciliados representaram as unidades elementares do estudo. Em cada unidade domiciliar foi aplicado um questionário epidemiológico para se obter informações sobre indicadores que favorecem a disseminação da doença no segmento peridomiciliar. 0 diagnóstico sorológico 
para DC em cães foi baseado em três métodos (RIFI, ELISA e HAI), sendo consideradas positivas aquelas amostras que apresentassem pelo menos dois testes reagentes e ausência de reatividade cruzada. Para zona rural do município, a prevalência de cães sororreagentes para T. cruzi por estrato amostral foram: Norte $6,05 \%$, Sul 3,59\% e Oeste 2,97\%, correspondendo a uma prevalência em sua totalidade de $4,08 \%$. Os fatores de riscos (odds ratio, OR) evidenciados em análise multifatorial foram: tipo de parede $(\mathrm{OR}=2,59$ [1,24-5,4]), presença de armazém (OR=1,89 [1,31-3,0]), presença de galinheiros (OR=8,31 [1,29-61,7]), contato com animais $(\mathrm{OR}=9,11[1,12-73,9])$, contato com aves $(\mathrm{OR}=9,7$ $[1,81-52,83])$, triatomíneos capturados $(\mathrm{OR}=16,58[3,43$ 80,23]) e antropismo (OR=4,35 [1,36-14,0]. Diante dos resultados foi possível se obter informações inerentes à situação epidemiológica da Doença de Chagas ressaltando características biogeográficas da zona rural do semiárido paraibano, elevando a espécie canina e os fatores de risco evidenciados, em destaque ao contato com aves e ecótopos artificiais, operacionalizando indicadores a serem assistidos e considerados na cadeia de transmissão da doença na região.

TERMOS DE INDEXAÇÃO: Doença de Chagas, prevalência, fatores de risco, zona rural, peridomicílio, cães.

\section{INTRODUÇÃO}

A Doença de Chagas é uma antropozoonose parasitária causada por Trypanosoma cruzi, um protozoário cujo ciclo de vida inclui a passagem obrigatória por vários hospedeiros mamíferos, para os quais é transmitido pelos insetos vetores denominados triatomíneos (Argolo et al. 2008).

Atualmente são conhecidos diferentes ciclos epidemiológicos de T. cruzi, dentre esses, o ciclo doméstico e peridoméstico, bem explorado epidemiologicamente, onde participam o homem e outros animais, bem como triatomíneos domiciliares e peridomiciliares. Seu início ocorreu quando o homem passou a ocupar os ecótopos silvestres, em vivendas rurais, oferecendo abrigo e alimento abundante aos vetores, servido de elo entre o ciclo doméstico e silvestre, incluindo-se, dessa forma, junto aos animais domésticos, no ciclo epidemiológico da doença (Schmuñis 2000). Alguns animais apresentam-se naturalmente infectados por T. cruzi, sendo conhecidos como animais reservatórios. Os mamíferos domésticos cresceram gradativamente em participação como reservatórios, onde podem, eventualmente, ser acometidos clinicamente pela doença (Levine 1973).

Nesse contexto, devido à estreita relação dos cães com o homem, esses são considerados em alguns países, como os principais reservatórios domésticos no ciclo peridomiciliar da infecção humana, porém com um papel na epidemiologia da doença de Chagas ainda a ser confirmada. Contudo, sabe-se que assume uma importante sentinela da doença em uma determinada região (Souza 2007). Dessa forma, investigações acerca de infecções em cães por T. cruzi necessitariam ser incluídas na saúde pública, visto ser essa doença comum em áreas rurais, com aumento dos relatos em áreas urbanas e grande variabilidade epidemiológica (Barr et al. 1991, Gürtler et al. 2007, Rosypal et al. 2007).
Alguns estudos têm sido feitos para estabelecer a relação entre fatores ambientais e os riscos de transmissão de T. cruzi. Um maior conhecimento destes fatores de risco é necessário para o desenvolvimento de apropriadas estratégias de intervenção em áreas endêmicas (Dias et al. 2000, Black et al. 2007). Alguns fatores de risco de interesse são os tipos de materiais usados na construção do telhado, paredes e piso da casa; a presença de palmeiras e mata ou floresta perto da casa; acúmulo de entulho ou lixo na área peridomiciliar; eletricidade; grau de escolaridade; ocupação ou emprego dos habitantes; história de transfusão sanguínea; presença de animais no domicílio ou no peridomicílio também podem estar associadas à infecção chagásica (Carneiro et al. 2001). Para tanto, é necessário o entendimento de como as variáveis ambientais e os fatores de risco interagem, no que se refere à infecção em cães, para que se possam operacionalizar medidas de desestabilização dos indicadores que interpõe sua infecção. Desta forma, o presente estudo teve como objetivo determinar os aspectos epidemiológicos que envolvem a Doença de Chagas canina e identificar os principais fatores de risco da enfermidade no semiárido paraibano.

\section{MATERIAL E MÉTODOS}

O estudo foi conduzido na zona rural do município de Patos, semiárido paraibano. Para estimativa da prevalência da doença de Chagas canina, a exploração da área estudada, seguiu critérios de estratificação baseada na atenção básica municipal, por meio de estudo amostral constituída de três estratos amostrais (Norte, Sul e Oeste) e aglomerados sistemáticos em um estágio, com intuito de obter uma amostragem uniforme da área rural. Na unidade de estágio, sorteou-se de forma aleatória simples sem reposição, um número pré-estabelecido de casas por estrato amostral da zona rural, sendo o número de casas sorteadas por estrato amostral determinada pela fórmula para amostras simples aleatórias, propostas por Thrusfield (2007), considerando uma prevalência esperada de $50 \%$, nível de significância de $5 \%$ e erro amostral de $5 \%$ (Quadro 1), representando $\pm 23 \%$ por estrato. Nas casas contempladas, além da investigação epidemiológica, foram realizadas coletas de sangue de todos os cães domiciliados, sem pré-requisitos quanto à idade, sexo ou raça.

Os testes sorológicos foram realizados no Laboratório municipal de Patos-PB, laboratório central de saúde pública da Paraíba (Lacen/PB), laboratório de Biologia Molecular (BIOMOL/UFCG) e no laboratório de referência em Taxonomia e diagnóstico de reservatórios silvestres das leishmanioses (Instituto Oswaldo Cruz/ RJ). A metodologia utilizada para o sorodiagnóstico de infecção por Trypanosoma cruzi nos cães foi baseada em três técnicas: (i) Reação de imunofluorescência indireta (RIFI) através de pesquisa de anticorpos séricos (IgG ant-T. cruzi), segundo Camargo

Quadro 1. Dados censitários das casas amostradas, por estrato amostral, da zonal rural do município de Patos, semiárido paraibano

\begin{tabular}{lccc}
\hline $\begin{array}{c}\text { Estratos } \\
\text { amostrais }\end{array}$ & $\begin{array}{c}\text { Total de } \\
\text { casas }^{\text {a }}\end{array}$ & $\begin{array}{c}\text { Casas } \\
\text { amostradas }\end{array}$ & $\begin{array}{c}\text { Total de } \\
\text { cães }\end{array}$ \\
\hline Norte & 358 & 83 & 99 \\
Sul & 575 & 133 & 167 \\
Oeste & 334 & 78 & 101 \\
Total & 1267 & 294 & 367
\end{tabular}

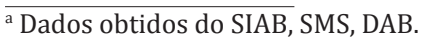


(1966). Os soros foram diluídos serialmente e um proporção decrescente de $2 x$ (1:10-1:320) e testados com antígenos totais de Trypanosoma cruzi. 0 ponto de corte para os títulos sorológicos foi de 1:20; (ii) ELISA (Enzyme-Linked Immunoabsorbent Assay), Nunc-Immuno Plates, MaxiSorp, Nalge Nunc International Corporation). Placa com antígeno sensibilizado na concentração de 2,4 $\mu \mathrm{g} / \mathrm{mL}$, com diluição amostra/conjugado 1:40.000, revelação (tetrametilbenzidina $+\mathrm{H}_{2} \mathrm{O}_{2}$ em solução diluente) e bloqueio da reação com $\mathrm{H}_{2} \mathrm{SO}_{2}$. Os controles foram estabelecidos a partir de cães sabidamente infectados e não infectados. (iii) Hemaglutinação indireta (Analisa CHAGAS-HAI), a partir determinação qualitativa e semi-quantitativa de anticorpos anti-T. cruzi no soro, pela aglutinação de eritrócitos estabilizados com componentes antigênicos do Trypanosoma cruzi altamente purificados, adaptados com controles caninos. Foram consideradas positivas para DC aquelas amostras que apresentassem pelo menos dois testes reagentes (Ministério da Saúde 2006), sendo o RIFI o teste "Gold standard" e ausência de reatividade cruzada para Leishmania infantum chagasi. A análise de concordância entre os testes foi determinada pelo teste Kappa e, a obtenção dos índices de sensibilidade, especificidade, valores preditivos positivo e negativo (VPP e VPN) e a análise dos testes associados em paralelo WIN EPISCOPE 2.0 (Thrusfield 2007).

Para investigação de reações cruzadas e co-infecções com $L$. infantum chagasi, foram realizados Reação de imunofluorescência indireta (RIFI) (Kit - Leishmaniose Visceral Canina Biomanguinhos), ELISA - (Kit EIE Leishmaniose Visceral Canina Biomanguinhos) e o Teste Imunocromatográfico DPP (Dual Path Plataform) Leishmaniose Visceral Canina (Biomanguinhos).

Em cada casa amostrada, além das coletas de sangue foi aplicado questionário epidemiológico estruturado, elaborado para se obter informações dos entrevistados sobre parâmetros e fatores que favorecessem a disseminação da doença, tais como indicadores sociais e econômicos (condições do domicílio e saneamento, escolaridade, saúde e renda familiar), indicadores dos fatores de risco da Doença de Chagas (variáveis ambientais, situação entomológica e aspectos relacionados sanidade do cão) e indicadores do grau de conhecimento sobre a Doença de Chagas. Para a análise de possíveis fatores de risco associados à condição do animal positivo, foram utilizados os dados obtidos por meio dos questionários epidemiológicos aplicados nas residências contempladas, desenvolvidas em duas etapas: análise univariada e análise multivariada. $\mathrm{Na}$ análise univariada, cada variável independente foi cruzada com a variável dependente (cão soropositivo para $T$. cruzi). As que apresentaram um valor de $\mathrm{P} \leq 0,2$ pelo teste de qui-quadrado ou teste exato de Fisher (Zar 1999), quando indicado, foram selecionadas e oferecidas para a análise multivariada, utilizando-se a regressão logística múltipla (Hosmer \& Lemeshow 2000). 0 ajuste do modelo final foi verificado com o teste de Hosmer e Lemeshow, pelo qual um valor de $p>0,05$ indica que $o$ modelo está ajustado. 0 nível de significância adotado na análise múltipla foi de $5 \%$. Todas as análises foram realizadas com os programas SPSS 13.0 for Windows e BioEstat versão 5.0 e WIN EPISCOPE 2.0. O presente trabalho foi aprovado pelo comitê de ética do Centro de Saúde e Tecnologia Rural da Universidade Federal de Campina Grande, sob o protocolo CEP 53/2012.

\section{RESULTADOS}

A soroprevalência da Doença de Chagas canina obtida nesta pesquisa foi de $4,08 \%$. 0 estrato amostral com maior número de registros de reagentes para Trypanosoma cruzi foi o Norte, com uma prevalência de 6,06\%, seguida pelo Sul e Oeste, respectivamente (Quadro 2). Condições de reatividade cruzada entre Leishmania infantum chagasi e T. cruzi,
Quadro 2. Prevalência de cães sororreagentes para Doença de Chagas na zona Rural do município de Patos, segundo estratos amostrais, no Estado da Paraíba

\begin{tabular}{|c|c|c|c|c|c|c|c|c|}
\hline \multirow[t]{3}{*}{ Estratos } & \multicolumn{8}{|c|}{ Sorologia - Trypanosoma cruzi } \\
\hline & \multicolumn{2}{|c|}{ RIFI+ELISA } & \multicolumn{2}{|c|}{ RIFI+HAI } & \multicolumn{2}{|c|}{$\mathrm{RIFI+ELISA+HAI}$} & \multicolumn{2}{|c|}{ Total } \\
\hline & $\mathrm{N}$ & $(\%)$ & $\mathrm{N}$ & $(\%)$ & $\mathrm{N}$ & $(\%)$ & $\mathrm{N}$ & $\%$ \\
\hline Norte & 1 & 1,01 & 3 & 3,03 & 2 & 2,02 & 6 & 6,06 \\
\hline Sul & 2 & 1,19 & 2 & 1,19 & 2 & 1,19 & 6 & 3,59 \\
\hline Oeste & 2 & 1,98 & 1 & 0,99 & 0 & 0 & 3 & 2,97 \\
\hline Total & 5 & 1,36 & 6 & 1,63 & 4 & 1,08 & 15 & 4,08 \\
\hline
\end{tabular}

considerando os métodos de sorodiagnóstico empregados, em sua totalidade foi registrada em $3,8 \%$ das amostras, sendo a RIFI o método que mais conduziu tal condição entre tripanossomatídeos $(\approx 25 \%)$. 0 índice Kappa mostrou concordância "moderada" entre o RIFI e ELISA e, concordância "boa" entre RIFI e HAI para as amostras de T. cruzi. 0 teste ELISA apresentou sensibilidade de $76 \%(74,6-99,0)$ e especificidade de $92 \%(86,5-95,1)$ com VPP e VPN de $45 \%$ e $96 \%$, respectivamente, enquanto o HAI apresentou sensibilidade de $82 \%(72,4-87,3)$ e especificidade de $95 \%$ $(87,5-98,0)$ com VPP e VPN de $76 \%$ e $97 \%$, respectivamente. A análise estatística dos testes combinada em paralelo entre o (1) RIFI e ELISA, (2) RIFI e HAI e, (3) ELISA e HAI revelaram uma sensibilidade e especificidade de: (1) $68 \%$ e $97 \%$, (2) 79\% e 98\% e, (3) 76\% e 94\%, respectivamente. $\mathrm{Na}$ análise univariada, as variáveis mais associadas à ocorrência de reatividade sorológica para T. cruzi em cães foram saneamento básico, contato com animais, contato com aves, contato com felinos, presença de triatomíneos, triatomíneos capturados, antropismo, tipo de parede, presença de curral, armazém e galinheiros. Na análise multivariada, as variáveis apontadas como fatores de risco pela regressão logística múltipla foram tipo de parede, presença de armazém e galinheiro, contato com animais, contato com aves, barbeiros capturados e antropismo (Quadro 3). 0 modelo final apresentou bom ajuste (teste de Hosmer e Lemeshow: qui-quadrado $<0,001 ; p=1,00$ ).

Quadro 3. Fatores de risco (odds ratio) para Doença de Chagas canina na zona rural do município de Patos no semiárido paraibano, estimados por regressão logística múltipla

\begin{tabular}{lccc}
\hline Fator de risco & Odds ratio & IC (95\%) & $\mathrm{p}$ \\
\hline Tipo de parede - Taipa & 2,59 & {$[1,24-5,4]$} & 0,01 \\
Armazém >50 metros & 1,98 & {$[1,31-3]$} & 0,0012 \\
Galinheiro - entre & 8,31 & {$[1,29-61,7]$} & 0,035 \\
10 e 50 metros & & & \\
Contato com animais & 9,11 & {$[1,12-73-9]$} & 0,038 \\
Contato com aves & 9,7 & {$[1,81-52,83]$} & 0,008 \\
Triatomíneo capturado & 16,58 & {$[3,43-80,23]$} & 0,0005 \\
Antropismo & 4,35 & {$[1,36-14,0]$} & 0,013
\end{tabular}

\section{DISCUSSÃO E CONCLUSÕES}

A região nordeste ocupa acentuada importância no contexto epidemiológico da Doença de Chagas no Brasil, sendo apontada como a segunda em número de infectados e de índices de infestação por triatomíneos nos inquéritos nacionais de prevalência e distribuição de vetores (Dias et al. 2000). Em inquéritos epidemiológicos envolvendo se- 
res humanos e animais, em cidades predominantemente rurais, os animais domésticos aparecem como importante fator de risco na transmissão doméstica de T. cruzi (Silva 2002).

Nesta pesquisa, a prevalência sorológica de cães naturalmente infectados por Trypanosoma cruzi (4,08\%), está abaixo daquela diagnosticada em área rural do Mato Grosso do Sul $(22,7 \%)$ através de três métodos de sorodiagnóstico (Souza et al. 2009), próximo da soroprevalência registrada em outra área do mesmo estado (3\%) pela técnica de IFI (Silva 1979) e, acima da prevalência obtida por Silva (2002) $(0,3 \%)$ em estudo conduzido em zona urbana na cidade de Porto Alegre/RS. Índices bem superiores ao deste estudo foram obtidos por Lauricela et al. (1989) (34\%) e Montenegro et al. (2002) $(27,7 \%)$ em pesquisa sobre a prevalência de cães chagásicos em áreas rurais da Argentina e do Chile, respectivamente. No entanto, na região Nordeste, pesquisa desenvolvida em áreas rurais do semiárido nordestino (Patos, Teixeira/PB e Caicó/RN) identificou a participação do cão como um potencial reservatório no ciclo epicemiológico do T. cruzi, com uma prevalência sorológica e parasitológica de $8,8 \%$ e 5,8\%, respectivamente, entretanto, com um papel ainda a ser esclarecido na transmissão para humanos (Santana 2011), diante do desconhecimento das variáveis que interpõe sua infecção e das características filogenéticas do parasita vigentes na região. No entanto, os índices de prevalência descritos anteriormente não conferem um efeito comparativo consistente, em virtude da não concordância entre os estudos no que se refere aos testes diagnósticos e metodologias de exploração empregadas. Valendo ressaltar a importância do emprego de técnicas diagnósticas combinadas, na melhoria da sensibilidade do teste em pesquisas de natureza epidemiológica.

Em toda área rural estudada, os cães parecem estar expostos ao ciclo de transmissão e T. cruzi, condição essa reforçada pelo registro de cães positivos em todos estratos amostrais. No entanto foi possível observar animais sororreagentes para T. cruzi e Leishmania infantum chagasi. Este fato configura a sobreposição do ciclo de transmissão desses parasitas no âmbito peridomiciliar. Por esse motivo, o diagnóstico canino para T. cruzi deve sempre ser avaliado com cautela, pela possibilidade de reação cruzada $\operatorname{com} L$. infantum chagasi ou situações de co-infecção entre parasitas, sendo esta última evidenciada através de ensaios moleculares.

Em relação aos fatores de risco, das sete variáveis validadas pela análise de regressão logística múltipla, todas, com exceção apenas dos triatomíneos capturados que constitui o vetor de transmissão do parasita, estão inseridas como indicadores de manutenção do ciclo peridomiciliar de T. cruzi (Foratine et al. 1979).

Os triatomíneos capturados apontados como fator de risco para infecção canina [odds ratio - 16,5] (Fig.1), representam uma contundente plausibilidade biológica diante do seu papel no ciclo epidemiológico da doença. A presença de triatomíneos domiciliares e peridomiciliares destacada em análise univariada, embora não considerada neste estudo como um fator de risco pela análise de regressão logística múltipla, merece ser destacada diante da sua alta

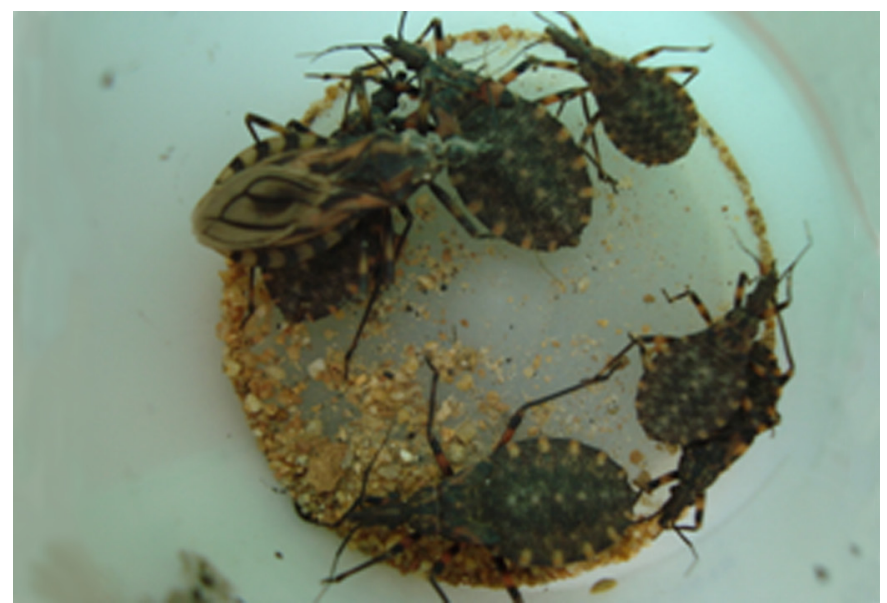

Fig.1. Triatomíneos capturados em segmento peridomiciliar.

frequência em $63 \%$ dos domicílios explorados e da prevalência de aproximadamente $50 \%$ dentre os caninos sororreagentes para T. cruzi.

Das espécies de triatomíneos capturadas ( $\mathrm{n}=24), 87,5 \%$ $(\mathrm{n}=21)$ foram as espécies Triatoma brasiliensis e 12,5\% $(\mathrm{n}=3)$ Triatoma pseudomaculata. Essas duas espécies em particular tem merecido atenção especial, diante da insatisfatória resposta ao controle químico, pela frequência e persistência da infestação domiciliar e peridomiciliar, pelo fato de colonizarem ecótopos artificiais peridomiciliares e a habitação e, pela alta densidade dessas espécies no ambiente silvestre e sua grande valência ecológica e ecletismo alimentar (Silveira et al. 2001). No entanto, novas medidas de controle e vigilância dessas espécies, associadas a um adequado manejo peridomiciliar, devem ser articuladas pelos órgãos responsáveis, dada a ineficiência comprovada baseada apenas no controle químico.

Neste contexto, o antropismo também destacado como fator de risco nesta pesquisa [odds ratio - 4,35] (Fig.2), observado em $12,8 \%$ das unidades domiciliares exploradas, demonstra que as atividades agropecuárias e desmatamento para exploração da madeira no semiárido paraibano, induzem a modificações microclimáticas e de oferta alimentar dos triatomíneos conduzindo a dispersão, adaptação e ocupação das moradias e anexos, tidos agora como novos

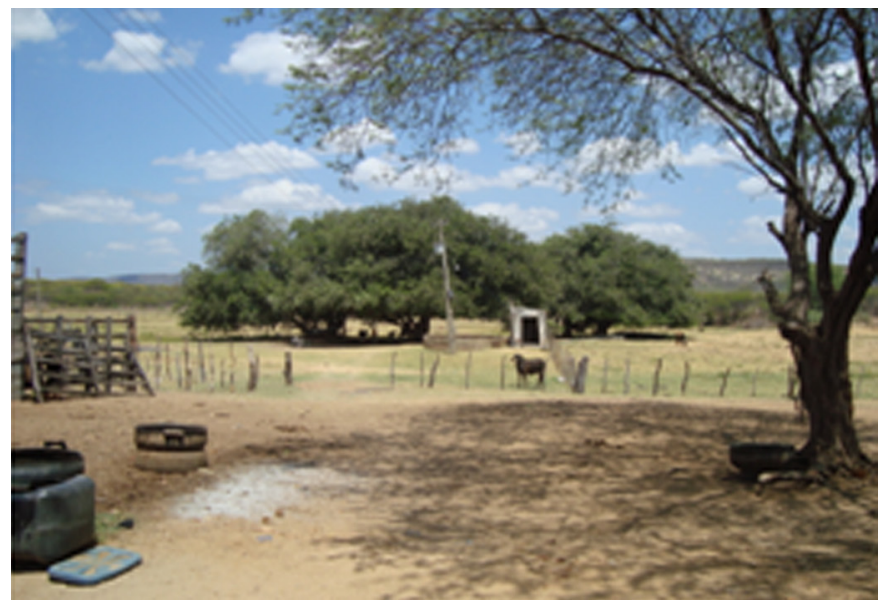

Fig.2. Antropismo. 


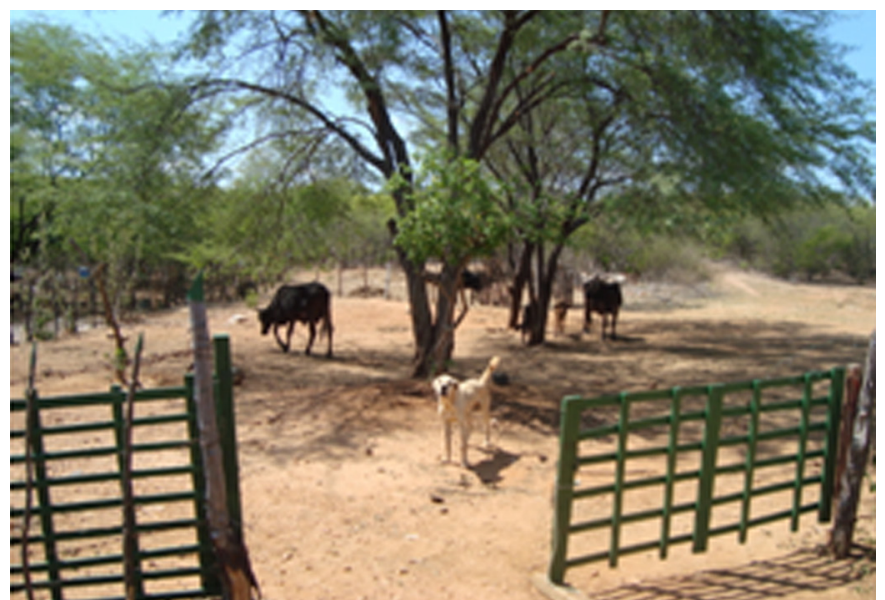

Fig.3. Contato com animais.

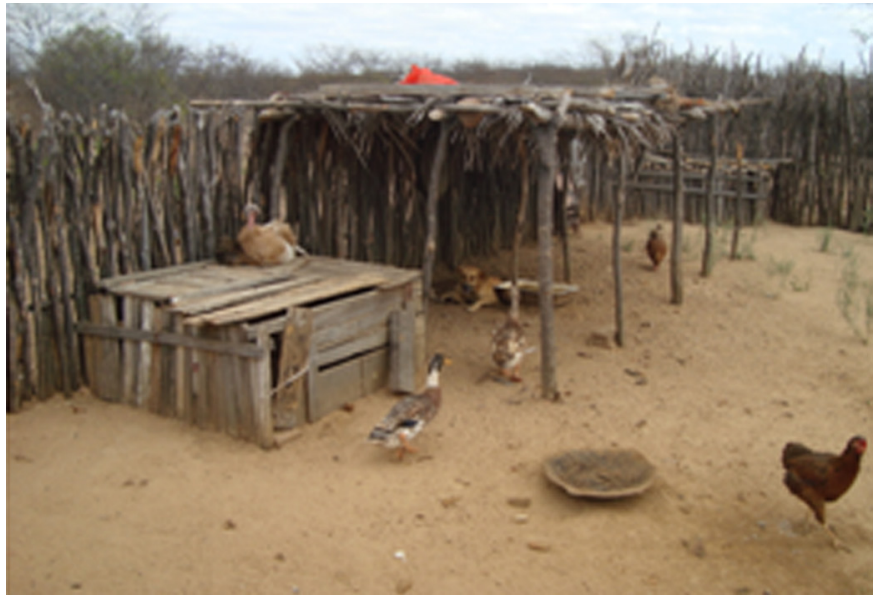

Fig.4. Contato com aves.

nichos ecológicos, antropizadas pela oferta de alimento (animais peridomiciliados e o homem) e abrigo (Foratine et al. 1979, Mendes et al. 2008). Desta forma, a criação de modelos que incluam os animais peridomiciliados, particularmente o cão, pela proximidade com o homem, permite a elaboração de novas ferramentas de predição e vigilância em focos naturais, artificiais e antropinizados de forma dinâmica e espacial (Romanã et al. 2003).

Outra condição apontada como fatores de risco foram o contato com animais peridomiciliados e domiciliados [odds ratio - 9,11] e com aves [odds ratio - 9,7](Fig.3 e 4). Esses indicadores evidenciam que a presença de animais no segmento peridomiciliar, principalmente as aves (galinhas, patos ou gansos), podem mediar infestações domiciliares por triatomíneos, fornecendo oferta alimentar, comportando-se como uma variável de manutenção do ciclo do parasita e de potencial infectividade para cães e humanos em áreas rurais do semiárido paraibano (Enger et al. 2004, Walter et al. 2007). Dados publicados por Dias et al. (2002) e Walter et al. (2007) elevam como fatores de risco a presença de animais domésticos na infestação peridomiciliar e consequentemente altos riscos de transmissão e infecção por T. cruzi. Por outro lado, um estudo realizado no noroeste da Argentina demonstrou que a presença de cães, gatos e galinhas pode diminuir tanto a infestação domiciliar por vetores, quanto a contaminação destes vetores por T. cruzi (Gurtler et al. 1998), contudo, esta última consideração, pode ser aplicada apenas a regiões onde há presença de triatomíneos com regime domiciliar, portanto, não enquadrada a este estudo, diante do hábito peridomiciliar dos triatomíneos vigentes na região do semiárida paraibano.

Atrelado ao fator de risco "contato com aves", encontra-se outro fator relacionado na análise multifatorial, a presença de galinheiro [odds ratio - 8,31] (Fig.5), que corresponde a um ecótopo artificial comum em áreas rurais da região semi-árida paraibana. Neste estudo obteve-se uma prevalência deste anexo peridomiciliar em 69\% das casas exploradas. A presença de galinheiros confere uma grande importância epidemiológica da Doença de Chagas na região, uma vez que a maioria dos triatomíneos apresentam hábitos exclusivamente silvestres, mesmo que sejam encontrados dentro das casas atraídos provavelmente pela luz, não conseguem se alimentar do sangue neste ambiente, onde passam a buscar ecótopos artificiais que os associam ao ambiente silvestre, passando a alimentar-se de diferentes hospedeiros peridomiciliares (Steindel et al. 1994). Tais considerações descritas anteriormente, se aplicam à presença do armazém (Fig.6) [odds ratio - 1,98], também apontado como fator de risco para Doença de Cha-

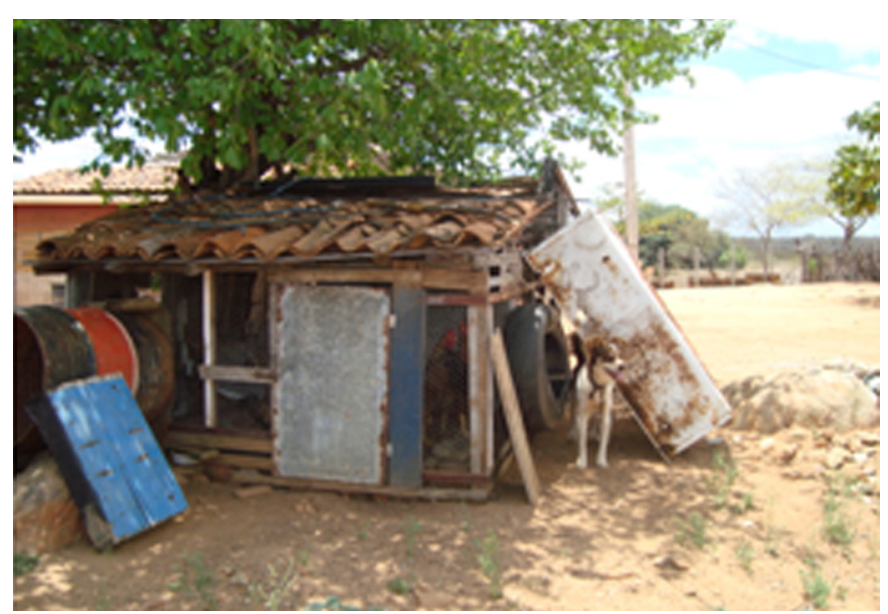

Fig.5. Galinheiro - entre 10 e 50 metros de distância da unidade domiciliar.

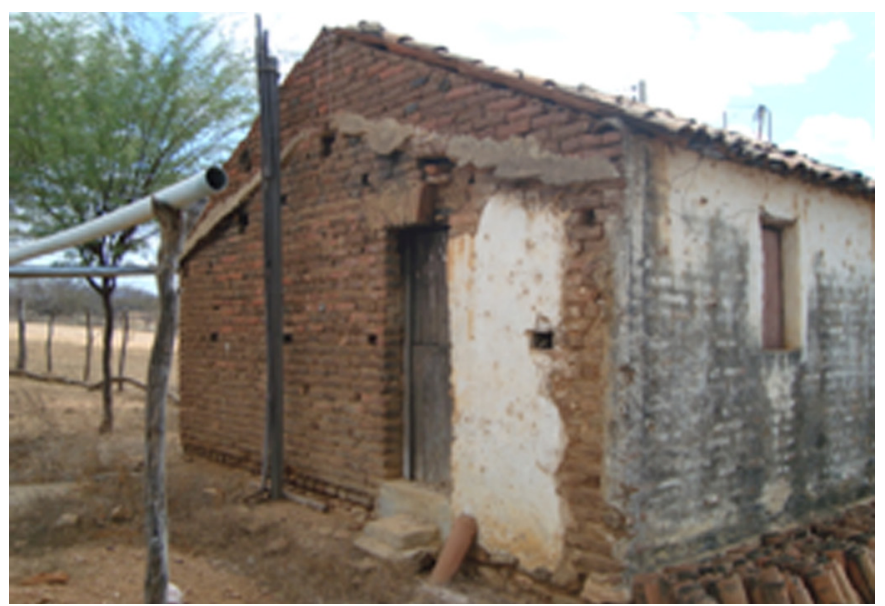

Fig.6. Armazéns. 


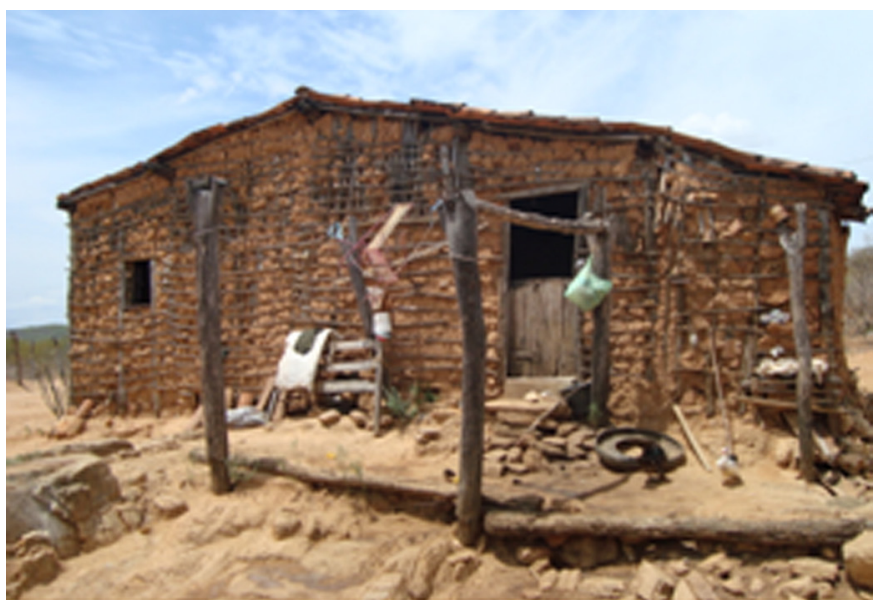

Fig.7. Tipo de parede - Taipa.

gas canina nesta pesquisa. Os armazéns são considerados no âmbito epidemiológico da doença de Chagas um anexo peridomiciliar, com características semelhantes aos ecótopos artificiais.

Em relação às características da unidade domiciliar, paredes de taipa sem reboco (Fig.7) apesar de representar apenas $10 \%$ das casas pesquisadas, apresentaram cães com sorologia positiva para T. cruzi em $50 \%$ dos que ali residiam, sendo considerado de fato ainda consistente fator de risco para espécie humana e canina [odds ratio - 2,59]. Uma alta frequência de habitações de alvenaria com reboco foi registrada neste estudo, tal mudança provavelmente ocorreu devido às melhorias habitacionais desenvolvidas pelo Programa de Controle da Doença de Chagas (PCDCh) e às das campanhas de controle à Doença de Chagas, com finalidade de interromper a transmissão vetorial domiciliar da doença (Brasil 2005). Contudo, estudos tem demonstrado sorologia reativa para essa enfermidade em áreas rurais com habitações de alvenaria com reboco, todavia associações entre a moradia atual e a sororreatividade para doença de chagas necessitam de maiores esclarecimentos para sua fundamentação (Dias et al. 2002).

De acordo com os resultados obtidos neste estudo foi possível se obter informações inerentes à situação epidemiológica da Doença de Chagas ressaltando características biogeográficas da zona rural do semiárido paraibano, elevando a espécie canina a uma variável a ser assistida e considerada na cadeia de transmissão da doença. Os indicadores apontados como fatores de risco neste estudo, com destaque para o contato com aves e ecótopos artificiais, podem contribuir para o desenvolvimento de novas estratégias de controle, prevenção e desestabilização do ciclo epidemiológico da doença na região semi-árida. Pode-se perceber também que inquéritos sorológicos e a evidenciação de fatores de risco são importantes ferramentas no dimensionamento de variáveis que se sobrepõe ao ciclo de transmissão do parasita, ressaltando condições particulares em uma natureza variada de uma determinada região. Outro ponto que merece destaque foi a identificação de cães jovens infectados com menos de 12 meses de idade, o que mostra que não se trata de uma exposição antiga a $T$. cruzi, e que a transmissão ainda ocorre na área.
Agradecimentos.- Ao Laboratório de Referência em Taxonomia e Diagnóstico de Reservatórios Silvestres 278 das Leishmanioses (IOC/Fiocruz/ RJ) no nome da Dra. Ana Maria Jansen e da Dra. Samantha Cristina C. 279 Xavier, ao Laboratório Central de Saúde Pública da Paraíba (Lacen-PB), a Secretaria Municipal de Saúde 280 (Semusa-Patos/PB), ao Laboratório de Incubação BIOGENE-PE no nome de Paulo Paes de Andrade, ao 281 Laboratório de Doenças Parasitárias da USP e ao Prof. Sérgio Santos de Azevedo pelo auxílio estatístico 282 prestado nesta pesquisa.

\section{REFERÊNCIAS}

Argolo A.M., Felix M., Pacheco R. \& Costa J. 2008. Doença de Chagas e seus Principais Vetores no Brasil. Imperial Novo Milênio, PIDC, Instituto Oswaldo Cruz, Fundação Oswaldo Cruz, Rio de Janeiro, p.16-17.

Barr S.C., Dennis V.A. \& Klein T.R. 1991. Serologic and blood culture survey of Trypanosoma cruzi infection in four canine populations of southern Louisiana. Am. J. Vet. Res. 52(4):570-573.

Black C.L., Ocaña S., Riner D., Costales J.A., Lascano M.S., Davila S., Arcos-Teran L., Seed J.R. \& Grijalva M.J. 2007. Household risk factors for Trypanosoma cruzi seropositivity in two geographic regions of Ecuador. J. Parasitol. 93:12-16.

Brasil 2005. Consenso Brasileiro em Doença de Chagas. Ministério da Saúde. Revta Soc. Bras. Med. Trop. 38(3):3-29.

Camargo M.E. 1966. Fluorescent antibody test for the sorodiagnosis of American trypanosomiasis: technical modification employing preserved culture forms of Trypanosome cruzi in a slide test. Revta Inst. Med. Trop. 8(5):227-235.

Carneiro M., Moreno E.C. \& Antunes C.M.F. 2001. Nested case-control study in a serological survey to evaluate the effectiveness of a Chagas disease control programme in Brazil. Bull. World Health Organization 79(5):409-4414.

Dias J.C.P., Machado E.M.M., Fernandes A.L. \& Vinhaes M.C. 2000. Esboço geral e perspectivas da Doença de Chagas no Nordeste do Brasil. Cad. Saúde Públ. 16(2):13-34.

Dias J.C., Silveira A.C. \& Schofield C.J. 2002. The impact of Chagas disease control in Latin America: a review. Mem. Inst. Oswaldo Cruz 97(5):603-12.

Enger K.S., Ordoñez R., Wilson M.L. \& Ramsey J.M. 2004. Evaluation of risk factors for rural infestation by Triatoma pallidipennis (Hemiptera: Triatominae), a Mexican vector of Chagas Disease. J. Med. Entomol. 41(4):760-767.

Forattini O.P., Ferreira O.A., Silva R.E.O \& Rabello E.X. 1979. Aspectos ecológicos da tripanossomíase americana XV. Desenvolvimento, variação e permanência de Triatoma sordida, Panstrongylus megistus e Rhodnius neglectus em ecótopos artificiais. Revta Saúde Pública. 13:220-234.

Gürtler R.E., Cecere M.C., Lauricella M.A., Cardinal M.V., Kitron U. \& Cohen J.E. 2007. Domestic dogs and cats as sources of Trypanosoma cruzi infection in rural northwestern Argentina. Parasitol. 134(1):69-82.

Gurtler R.E., Cohen J.E., Cecere M.C., Lauricella M.A., Chuit R. \& Segura E.L. 1998. Influence of humans and domestic animals on the household prevalence of Trypanosoma cruzi in Triatoma infestans populations in north west Argentina. Am. J. Trop. Med. Hyg. 58(6):748-758.

Hosmer D.W. \& Lemeshow S. 2000. Applied Logistic Regression. John Wiley and Sons, New York. 375p.

Lauricella M.A., Sinagra A.J., Paulone I., Riarte A.R. \& Segura E.I. 1989. Natural Trypanosoma cruzi infection in dogs of endemic areas of Argentine Republic. Revta Inst. Med. Trop. 31:63-70.

Levine N.D. 1973. Protozoan Parasites of Domestic Animals and of man. $2^{\text {nd }}$ ed. Burgess Publishing Company, Mineapolis, p.54-58.

Mendes P.C., Lima S.C., Paula M.B.C., Souza A.A., Rodrigues E.A.S. \& Limongi J.E. 2008. Doença de Chagas e a distribuição espacial de triatomíneos capturados em Uberlândia, Minas Gerais, Brasil. Hygeia, Revta Bras. Geogr. Med. Saúde 3(6):176-204.

Ministério da Saúde 2006. Manual de Vigilância e Controle da Leishmaniose Visceral, $2^{\underline{a}}$ ed. Secretaria de Vigilância em Saúde, Ministério da Saúde, Brasília, DF. 122p. Disponível em <http://www.saude.to.gov.br/ index.php?option=com_jdownloads\&Itemid=999999\&view=viewdown load\&catid $=186 \&$ cid $=1045>$ 
Montenegro V.M., Jiménez M., Pinto Dias J.C. \& Zeledón R. 2002. Chagas disease in dogs from endemic areas of Costa Rica. Mem. Inst. Oswaldo Cruz 97:491-494

Romaña C., Emperaire L. \& Jansen A.M. 2003. Enfoques conceptuales y propuestas metodológicas para el estudio de las interacciones entre el médio ambiente y la salud: aplicación a un programa de investigación sobre La tripanosomiasis americana. Cad. Saúde Pública 19(4):945-953.

Rosypal A.C., Cortés-Vecino J.A., Gennari S.M., Dubey J.P., Tidwell R.R. \& Lindsay D.S. 2007. Serological surgey of Leishmania infartum and Trypanosoma cruzi in dogs from urban areas of Brazil and Colombia. Vet. Parasitol. 149:172-177.

Santana V.L. 2011. Doença de Chagas em cães em região do semiárido nordestino. Dissertação de Mestrado em Medicina Veterinária. Centro de Saúde e Tecnologia Rural, Universidade Federal de Campina Grande, Patos, PB. 68p.

Schmuñis G.A. 2000. A tripanosomíase americana e seu impacto na saúde pública da Américas, p.84-98. In: Brener Z., Andrade Z. A. \& Barral-Neto M. (Eds), Trypanosoma cruzi e Doença de Chagas. $2^{\underline{a}}$ ed. Guanabara Koogan, Rio de Janeiro.

Silva L.S. 2002. Prevalência de soropositvos para doença de chagas em uma amostra da população de cães domiciliados da cidade de Porto Alegre. Dissertação de Mestrado em Cardiologia e Ciências Cardiovasculares, Faculdade de Medicina, Universidade Federal do Rio Grande do Sul, Porto Alegre, RS. 80p.

Silva R.P. 1979. Estudo sobre Trypanosoma (Schizotrypanum) cruzi em área de Mato Grosso do Sul: casos humanos, reservatórios e transmissores. Tese de Doutorado, Instituto de Biociências, Universidade de São Paulo, São Paulo, SP. 83p.

Silveira A.C., Vinhaes M.C., Lira E. \& Araújo E. 2001.0 controle de Triatoma brasiliensis e Triatoma pseudomaculata. Organização Pan-Americana da Saúde, Brasília, p.21-27.

Souza A.I. 2007. Estudo clínico da infecção natural por Trypanosoma cruzi em cães residentes em uma área rural de mato grosso do sul, Brasil. Dissertação de Mestrado em Ciências Agrárias e Veterinárias, Universidade Estadual Paulista "Júlio Mesquita Filho", Jaboticabal, SP. 78p.

Souza A.I., Oliveira T.M.F.S., Machado R.Z. \& Camacho A.A. 2009. Soroprevalência da infecção por Trypanosoma cruzi em cães de uma área rural do Estado de Mato Grosso do Sul. Pesq. Vet. Bras. 29(2):150-152.

Steindel M., Toma H.K., Carvalho Pinto C.J., Grisard E.C. \& Schlemper Jr B.R. 1994. Colonização de ecótopos artificiais por Panstrongylus megistus na Ilha de Santa Catarina, Santa Catarina, Brasil. Revta Inst. Med. Trop. 36 (1):43-50

Thrusfield M. 2007. Veterinary Epidemiology. $3^{\text {rd }}$ ed. Blackwell Science, Oxford. 610p.

Walter A., Kasten-Lozano F., Bosseno M.F., Luna C.E.M., Baunaure F., Phélinas P., Megallon-Gastelun E. \& Breniére S.F. 2007. Peridomicilary habitat and risk factors for Triatoma infestation in a rural community of the Mexican Occident. Am. J. Trop. Med. Hyg. 76(3):508-515.

Zar J.H. 1999. Biostatistical Analysis. $4^{\text {th }}$ ed. Prentice Hall, Upper Saddle River. 663p. 